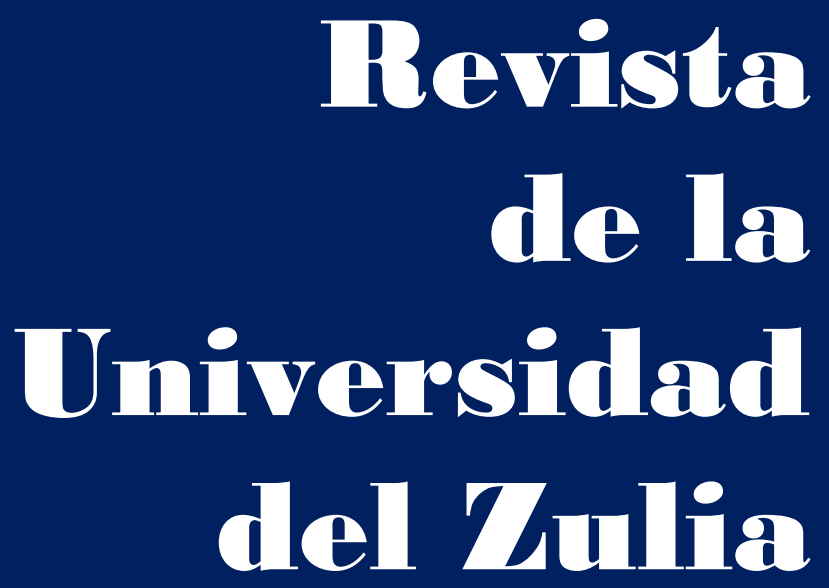

Fundada en 1947

por el Dr. Jesús Enrique Lossada

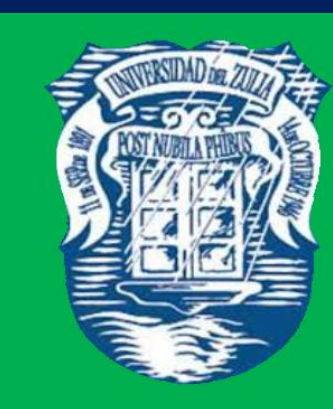

Ciencias del

Agrad,

Ingemiería

y Tecinología

\section{Aกัต 13 No $\mathbf{3 6}$} Enero - Abril 2022

Tercera Épaca

Maracailbo-Venezuela 


\title{
Análisis proximal y rendimiento de cinco especies nativas con valor forrajero
}

\author{
Manuel Oliva * \\ Jegnes Benjamín Meléndez-Mori** \\ Jorge Luis Maicelo-Quintana*** \\ Manuel Emilio Milla-Pino**** \\ Santos Leiva*****
}

RESUMEN

El objetivo del presente estudio fue evaluar el establecimiento, crecimiento, rendimiento y valor nutritivo de cinco especies nativas con valor forrajero (Cenchrus clandestinus, Philoglossa mimuloides, Philoglossa sp., Trifolium dubium y Trifolium repens). El experimento se realizó en el distrito de Molinopampa (Perú), donde se instalaron parcelas de $30 \mathrm{~m}^{2}$. La altura de planta se midió mensualmente hasta los 90 días. El material se cortó a nivel del suelo, se pesó, se secó y se determinaron los rendimientos de forraje verde y materia seca. El valor nutricional de las muestras se realizó siguiendo el procesamiento de la AOAC. Los resultados muestran que el porcentaje de establecimiento más alto fue alcanzado por T. repens $(89,51 \%)$. La altura de planta mostró que $P$. mimuloides y Philoglossa sp. registraron su mayor crecimiento entre los días 30 y 60 después del establecimiento; y además fueron las especies con mayores niveles forraje verde $\left(7,46\right.$ y $\left.8,04 \mathrm{~kg} / \mathrm{m}^{2}\right)$ y materia seca ( 8,58 y $8,90 \mathrm{t} / \mathrm{ha})$. En términos de valor nutricional, T. dubium y C. clandestinus registraron los niveles más altos de proteína y fibra, respectivamente; mientras que las especies del género Philoglossa tienen mayor digestibilidad.

PALABRA CLAVE: ganado; recursos alimentarios; pastizal; recursos animales.

* Investigador del Instituto de Investigación para el Desarrollo Sustentable de Ceja de Selva (INDES-CES), Universidad Nacional Toribio Rodríguez de Mendoza (UNTRM), Chachapoyas 01001, Perú. ORCID: https://orcid.org/0000-00029670-0970.

** Instituto de Investigación para el Desarrollo Sustentable de Ceja de Selva (INDES-CES), Universidad Nacional Toribio Rodríguez de Mendoza (UNTRM), Chachapoyas 01001, Perú. ORCID: https://orcid.org/0000-0002-1489-7395. E-mail: jbenjamin@indes-ces.edu.pe (autor de correspondencia)

*** Instituto Nacional de Innovación Agraria (INIA), Lima, Perú. ORCID: https://orcid.org/0000-0001-9109-0504

**** Facultad de Ingeniería Civil, Universidad Nacional de Jaén, Cajamarca, Perú. ORCID: https://orcid.org/00000003-3931-9804

***** Instituto de Investigación para el Desarrollo Sustentable de Ceja de Selva (INDES-CES), Universidad Nacional Toribio Rodríguez de Mendoza (UNTRM), Chachapoyas 01001, Perú. ORCID: https://orcid.org/0000-0003-1710-1994

Recibido: 21/09/2021

Aceptado: 12/11/2021 


\section{Proximal analysis and performance of five native species with forage value}

ABSTRACT

The objective of this study was to evaluate the establishment, growth, performance and nutritive value of five native species with forage value (Cenchrus clandestinus, Philoglossa mimuloides, Philoglossa sp., Trifolium dubium, and Trifolium repens). The experiment was conducted in the district of Molinopampa (Peru), where $30 \mathrm{~m} 2$ plots were installed. Plant height was measured monthly until 90 days. The material was cut at ground level, weighed, dried and green forage and dry matter performances were determined. The nutritional value of the samples was made following AOAC processing. The results show that the highest percentage of establishment was achieved by T. repens (89.51\%). Plant height showed that P. mimuloides and Philoglossa sp. registered their highest growth between the 30th and 60th day after establishment, they were also the species with the highest levels of green forage $(7.46$ and $8.04 \mathrm{~kg} / \mathrm{m} 2)$ and dry matter ( 8.58 and $8.90 \mathrm{t} / \mathrm{ha}$ ). In terms of nutritional value, T. dubium and C. clandestinus recorded the highest levels of protein and fiber, respectively, while Philoglossa species have higher digestibility.

KEYWORDS: Cattle; Food resources; Grasslands; Animal resources.

\section{Introducción}

En la alimentación del ganado durante los últimos años se ha extendido el uso de especies forrajeras mejoradas. La popularización acelerada de especies herbáceas no nativas con valor forrajero se debe a su potencial para aportar altos niveles productivos y valor nutritivo (Villalobos y Arce, 2014). Sin embargo, la producción de carne y leche no sólo depende de la calidad del alimento, sino también del adecuado aprovechamiento del forraje y de la selección de la raza. En las regiones donde la ganadería es familiar, la mala gestión de los componentes (especies forrajeras y animales) pueden generar inconvenientes para la actividad al libre pastoreo, ya que casi siempre se desarrolla sin un criterio técnico, existiendo sobrepastoreo y sobrecarga animal (Alegre et al., 2019).

Las especies introducidas o mejoradas requieren de condiciones edafoclimáticas idóneas para expresar su potencial genético. Las condiciones estacionales particulares de cada región influyen significativamente sobre el desarrollo de los pastizales (Fernández et al., 2001). Esto 
implica que las variaciones de temperatura y los cambios en los patrones de lluvia pueden provocar un desbalance en los rendimientos, afectando la disponibilidad de los forrajes (Fernández et al., 2000; Primavesi y Primavesi, 2002). En este contexto, es importante que los ganaderos seleccionen especies aptas para el clima y suelo de cada zona (Primavesi y Primavesi, 2002; Clavero, 2013). Una alternativa importante se encuentra en la diversidad nativa, donde es posible encontrar especies adaptadas a las condiciones agroclimáticas del lugar, de alta productividad y gran valor nutricional (Fernández et al., 2001; Uvidia et al., 2013).

La introducción de especies puede ejercer una presión negativa sobre la diversidad de herbáceas forrajeras nativas (Dresseno et al., 2018). Las perturbaciones ocasionadas por las especies exóticas pueden tener graves consecuencias sobre la composición de la biodiversidad (Pedrini et al., 2018). La competencia por coexistir puede fragmentar el hábitat de los forrajes nativos (Bakker y Wilson, 2001), modificar las interacciones abiótico-bióticas (Godfree et al., 2017), y alterar la estructura y cantidad de nutrientes del suelo (Dresseno et al., 2018). Por ello, para asegurar la sostenibilidad de la ganadería es importante que las personas comprendan la importancia y el comportamiento de las especies nativas con valor forrajero.

Bajo esas premisas es fundamental el estudio de especies locales, dado que la limitada información dificulta la revaloración del potencial de las especies forrajeras nativas que predominan en las cuencas ganaderas de la región Amazonas (Perú) (Oliva et al., 2015a). Por lo anterior, el objetivo del presente estudio fue evaluar el establecimiento, crecimiento, rendimiento y el valor nutritivo de cinco especies nativas con valor forrajero, con posibles potencialidades para la actividad ganadera local.

\section{Materiales y métodos}

\section{1. Área de estudio}

El estudio se desarrolló en el distrito de Molinopampa ubicado en la provincia de Chachapoyas, región Amazonas, al norte de Perú; se ubica entre los 06¹'45" de latitud Sur y $77^{\circ} 38^{\prime} 15^{\prime \prime}$ de longitud Oeste, a una altitud sobre los $2400 \mathrm{~m}$ s.n.m. La zona se caracteriza por un clima ligeramente húmedo y templado cálido (sin estaciones climáticas diferencias), con una 
temperatura promedio anual de $14,5^{\circ} \mathrm{C}$ y precipitación promedio de $1200 \mathrm{~mm}$ /año (IIAP, 2007); entre los meses de noviembre a marzo se registra la mayor intensidad de lluvia.

\subsection{Diseño experimental}

El ensayo se dirigió bajo un diseño de bloques completos al azar conformado por cinco tratamientos (especies forrajeras nativas) y tres réplicas. Se establecieron parcelas experimentales de $30 \mathrm{~m}^{2}$, donde a partir de material vegetativo (brotación) se plantó cinco especies de forrajeras nativas: 1) Cenchrus clandestinus (Hochst. ex Chiov.) Morrone (Poaceae) (kikuyo), 2) Philoglossa mimuloides (Hieron.) H. Rob. \& Cuatrec. (siso lapacho), 3) Philoglossa sp. (siso menudo) (Asteraceae), 4) Trifolium dubium Sibth. (trebolillo) y 5) Trifolium repens L. (trébol blanco) (Fabaceae), distanciadas a $20 \mathrm{~cm}$ entre surcos y $10 \mathrm{~cm}$ entre plantas.

\subsection{Establecimiento, crecimiento y rendimiento}

Después de 30 días de plantación, el porcentaje de prendimiento (eficiencia de implantación) se determinó contando el número de plantas vivas respecto al número total de plantas instaladas. Además, durante los siguientes 90 días se evaluó su crecimiento (altura de la planta), para lo cual se seleccionaron al azar 30 plantas por tratamiento (10 plantas por repetición).

Para estimar el rendimiento, los muestreos se llevaron a cabo después de 90 días de la plantación (inicio de floración), para lo cual el forraje se cortó a nivel del suelo. La producción de forraje verde (FV) se determinó usando un cuadrante de $1 \mathrm{~m}^{2}$ y una balanza (precisión 0,1 g). La materia seca (MS) se calculó tomando $1 \mathrm{~kg}$ de forraje (muestra representativa y homogénea) que fue secado a $65^{\circ} \mathrm{C}$ durante 72 horas y luego pesado para estimar la MS por diferencia de peso.

\subsection{Análisis proximal}

Las muestras se secaron en una estufa a $65^{\circ} \mathrm{C}$ durante 24 h y luego se realizó el análisis proximal: humedad (H), proteína cruda (PC), extracto etéreo (EE), fibra cruda (FC) y cenizas (C) por el método descrito por la AOAC (AOAC, 1990), digestibilidad in vitro (DIVMS) mediante el método enzimático pepsina-celulasa (Clarke et al., 1982) y extracto libre de nitrógeno (ELN) mediante un cálculo por diferencia $[100-(\mathrm{H}+\mathrm{PC}+\mathrm{EE}+\mathrm{FC}+\mathrm{C})]$. 


\subsection{Análisis de datos}

Los resultados obtenidos fueron evaluados mediante un análisis de varianza con un $5 \%$ de significancia $(\alpha=0,05)$; asimismo, fueron sometidos a la prueba de comparaciones múltiples de Tukey $(\mathrm{p} \leq 0,05)$. Todos los datos fueron evaluados previamente usando la prueba ShapiroWilk y Levene para determinar la normalidad y homogeneidad de varianzas. Los datos se analizaron en el programa SPSS v.15 (SPSS Inc, 2006).

\section{Resultados}

\subsection{Establecimiento, crecimiento y rendimiento}

Durante el período de evaluación las especies no mostraron diferencias significativas en el prendimiento (\%). No obstante, se observó que T. repens $(89,51 \%)$ y T. dubium $(88,25 \%)$ presentaron una sobrevivencia numéricamente mayor, principalmente comparado con $P$. Mimuloides (78,98\%).

La altura de planta mostró una dependencia significativa de la edad de plantación, es decir, la mayor altura de planta se alcanzó a los 90 días después del establecimiento. Las especies P. mimuloides y Philoglossa sp. fueron quienes presentaron los valores más altos para altura de planta $\left(32,34\right.$ y $33,89 \mathrm{~cm}$ respectivamente) y rendimiento de forraje verde $\left(7,46\right.$ y $8,04 \mathrm{~kg} / \mathrm{m}^{2}$ respectivamente). Sin embargo, estas especies tuvieron los porcentajes más bajos de materia (11,50 y 11,07\% respectivamente) (Tabla 1). En contraste, T. dubium, C. clandestinus y T. repens alcanzaron menores alturas y rendimientos de forraje verde (Tabla 1). Los rendimientos de materia seca de P. mimuloides, Philoglossa y C. clandestinus fueron superiores a $8 \mathrm{t} / \mathrm{h}$ a, mientas que los rendimientos de T. dubium y T. repens fueron inferiores a $5 \mathrm{t} / \mathrm{ha}$ (Tabla $\mathrm{l}$ ).

\subsection{Análisis proximal}

El análisis de varianza y la prueba de comparaciones múltiples de los parámetros nutricionales mostraron diferencias altamente significativas $(\mathrm{P} \leq 0,01)$ entre las especies (Figura 1). Mediante el análisis de sus componentes nutricionales se observó que P. mimuloides presentó un mayor valor de humedad (7,09\%) y ELN (61,68\%), T. dubium generó mayores valores de proteína (25,68\%) y grasa (extracto etéreo) (2,18\%); C. clandestinus registró mayor valor de 
REVISTA DE LA UNIVERSIDAD DEL ZULIA. 3época. Año 13 N 36, 2022

Manuel Oliva et al. /// Análisis proximal y rendimiento de cinco especies nativas con valor forrajero, 114-125

DOI: http://dx.doi.org/10.46925//rdluz.36.08

fibra $(25,2 \%)$ y T. repens alcanzó mayores valores de ceniza (10,52\%). En cuanto a la digestibilidad, los valores más altos $(71,18$ - 73,27\%) se registraron en las especies del género Philoglossa.

Tabla 1. Altura de planta, forraje verde y materia seca de cinco especies forrajeras nativas.

\begin{tabular}{|c|c|c|c|c|c|c|}
\hline \multirow[t]{2}{*}{$\begin{array}{c}\text { Especie forrajera } \\
\text { nativa }\end{array}$} & \multicolumn{3}{|c|}{$\begin{array}{c}\text { Altura de planta }(\mathrm{cm}) \text { en } \\
\text { distintas edades de plantación } \\
\text { (días) }\end{array}$} & \multirow{2}{*}{$\begin{array}{l}\text { Forraje } \\
\text { Verde } \\
\left(\mathrm{kg} / \mathrm{m}^{2}\right)\end{array}$} & \multirow[t]{2}{*}{$\begin{array}{l}\text { Materia } \\
\text { Seca }(\%)\end{array}$} & \multirow{2}{*}{$\begin{array}{c}\text { Materia } \\
\text { seca } \\
\text { (t/ha) }\end{array}$} \\
\hline & 30 & 60 & 90 & & & \\
\hline Philoglossa mimuloides & $9,28^{a}$ & $27,92^{a}$ & $32,34^{a}$ & $7,46^{a}$ & $11,50^{b}$ & $8,58^{a}$ \\
\hline Trifolium dubium & $5,41^{b}$ & $14,87^{b}$ & $17,89^{b}$ & $2,52^{c}$ & $18,37^{\mathrm{ab}}$ & $4,63^{b}$ \\
\hline Philoglossa sp. & $9,01^{\mathrm{a}}$ & $27,82^{a}$ & $33,89^{a}$ & $8,04^{a}$ & $11,07^{b}$ & $8,90^{a}$ \\
\hline Cenchrus clandestinus & $7,56^{\mathrm{a}}$ & $15,74^{b}$ & $19,25^{b}$ & $4,20^{b}$ & $20,03^{a}$ & $8,38^{a}$ \\
\hline Trifolium repens & $9,58^{a}$ & 18,39 ab & $22,41^{b}$ & $2,76^{c}$ & $17,30^{a b}$ & $4,90^{b}$ \\
\hline
\end{tabular}

a,b,c medias con letras diferentes en una misma columna difieren entre sí, según la prueba Tukey $(\mathrm{p} \leq 0,05)$.

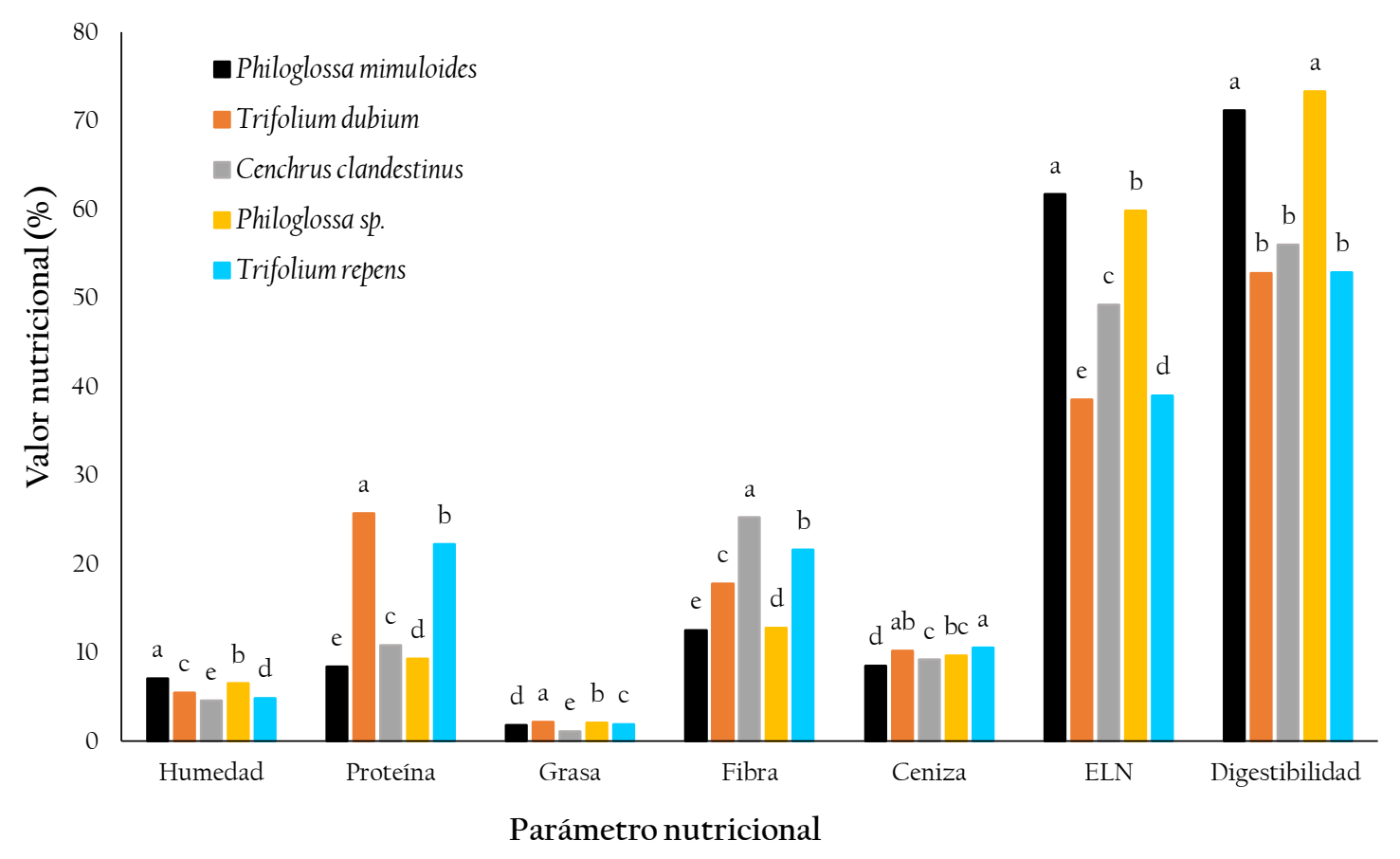

Figura 1. Valor nutricional registrado para cinco especies forrajeras nativas. 


\section{Discusión}

\subsection{Establecimiento, crecimiento y rendimiento}

En este estudio, la eficiencia de implantación en cuatro de las especies forrajeras superó el 80\%; y aunque las diferencias entre especies no fueron significativas, sus resultados muestran una capacidad de establecimiento que se debe continuar evaluando. Las especies nativas constituyen un recurso importante que puede ser estudiado y utilizado para lograr la cobertura de las áreas de pastoreo (Oliva et al., 2015b), ya que pueden presentar mejor adaptabilidad a las condiciones climáticas particulares de cada región. Las variables climáticas pueden cambiar significativamente a lo largo del año, incluso dentro de la misma área, por lo que se convierten en factores que inciden en el desarrollo del componente forrajero (Gandullo et al., 2013; Velasco et al., 2018). El análisis de la capacidad adaptativa de las especies muestra que el germoplasma puede ser explotado en la zona de estudio y sus áreas circundantes, y proporcionar forrajes para el desarrollo de la actividad ganadera local.

El análisis de la altura de la planta permitió constatar que esta variable (en todas las especies) mantuvo un incrementó constante a partir del día 30 después de plantación. Este comportamiento fue más pronunciado en las especies del género Philoglossa (Asteraceae) debido a su mayor tasa de crecimiento (0,38-0,4l cm/día, datos no mostrados), comparado con las otras especies (0,19-0,21 cm/día, datos no mostrados). Sin embargo, es importante tener en cuenta que la diferencia de altura comparado con las especies del género Trifolium está más relacionada con la morfología de la planta, debido a que estas mostraron un comportamiento y dinámica de crecimiento típicas de la familia de las leguminosas (Fabaceae) (Wachendorf et al., 2001); ya que la activación de las yemas vegetativas requiere un período de descanso más prolongado antes de que pueden iniciar su crecimiento. En general, la abundancia de estas especies las convierte en una importante alternativa para la producción de forraje en las praderas naturales de la región Amazonas (Oliva et al., 2015a; Oliva et al., 2019).

La evaluación del crecimiento del forraje (altura de la planta) es muy importante porque es un parámetro que presenta una relación proporcional con la acumulación de biomasa (Fernández et al., 2001). En este estudio se observó que las especies del género Philoglossa obtuvieron los valores de rendimiento más altos. Un resultado similar fue reportado por Oliva et 
REVISTA DE LA UNIVERSIDAD DEL ZULIA. 3르 época. Año 13 N³6, 2022

Manuel Oliva et al. /// Análisis proximal y rendimiento de cinco especies nativas con valor forrajero, 114-125

DOI: http://dx.doi.org/10.46925//rdluz.36.08

al. (2015b), donde especies de este género presentaron el mayor rendimiento en comparación con otras especies nativas. En cuanto a los niveles de materia seca, Pereira-Crespo et al. (2012) reportaron que el valor de las leguminosas del género Trifolium osciló entre 11 a 13 \%, siendo resultados similares a lo que este estudio registró para Philoglossa sp. y Philoglossa mimuloides, pero inferiores los registros de T. dubium, C. clandestinus y T. repens.

Es importante resaltar que tanto el crecimiento como la velocidad de recuperación (rebrote) de las pasturas está influenciada por factores como la acumulación de carbohidratos en sus estructuras vegetativas (Castro et al., 2013; Lemaire y Agnusdei, 2000), la especie y su potencial genético (Castro et al., 2013; Rodríguez et al., 2011; Uvidia et al., 2013). Además, se debe considerar a la temperatura, radiación y la precipitación como factores determinantes en la tasa de crecimiento y la calidad de las especies forrajeras de interés (Fernández et al., 2001; Wachendorf et al., 2001; Velasco et al., 2018).

\subsection{Análisis proximal}

Los valores de proteína y fibra de los forrajes indican que C. clandestinus (proteína: 12,55 \% y fibra: 17,62 \%), T. repens (proteína: 19,90 \% y fibra: 14,15\%) y T. dubium (proteína: 23,29 \% y fibra: $13,34 \%$ ) tienen un comportamiento normal para la zona (Oliva et al., 2015b), es decir, el nivel alcanzado es cercano, y puede sugerir que estos parámetros dependen de la especie a cultivar. Estos resultados muestran el potencial forrajero de las especies nativas. Además, las especies del género Trifolium también pueden ofrecer una fuente alternativa de nitrógeno para la producción de forraje, adicional a la aplicación de fertilizantes (Egan et al., 2017). Los niveles de ceniza, comprendidas en un rango de 8 a $11 \%$, son similares a los reportados para especies como Brachiaria decumbens Stapf y Cynodon nlemfuensis Vanderyst (Poaceae) (Combatt et al., 2015); determinación que es importante por estar relacionado con la disponibilidad de minerales en el forraje (Combatt et al., 2015). Respecto a la digestibilidad, los resultados obtenidos a excepción de la especie Philoglossa sp. fueron superiores a investigaciones realizadas con maní forrajero Arachis pintoi Krap. \& Greg. (Fabaceae), cuyos valores se comprendieron entre 55 a 67 \% (Godoy et al., 2012), similar resultado fue reportado para morera Morus alba L. (Moraceae), pues el nivel de NDT fue inferior a 60 \% (Boschini, 2006). No obstante, Estupiñán et al. (2007) y Godoy et al. 
REVISTA DE LA UNIVERSIDAD DEL ZULIA. 3르 época. Año 13 N³6, 2022

Manuel Oliva et al. /// Análisis proximal y rendimiento de cinco especies nativas con valor forrajero, 114-125 DOI: http://dx.doi.org/10.46925//rdluz.36.08

(2012) señalan que la digestibilidad de las pasturas varía según el estado vegetativo de la planta, siendo importante establecer el estado óptimo para su aprovechamiento.

\section{Conclusiones}

Las especies P. mimuloides y Philoglossa sp. obtuvieron los más altos valores de forraje verde $\left(7,46\right.$ y $\left.8,04 \mathrm{~kg} / \mathrm{m}^{2}\right)$ y materia seca $(8,58$ y $8,90 \mathrm{t} / \mathrm{ha})$. Asimismo, se ha comprobado que las especies influyen sobre el nivel de cada componente nutricional, siendo T. dubium la especie que presentó el mayor contenido de proteína. Las especies evaluadas presentan condiciones que pueden favorecer la sostenibilidad de la actividad ganadera en la zona de estudio, pero sus posibilidades de cultivo y la gestión equilibrada de cada especie dentro de los pastizales deben continuar evaluándose.

Agradecimientos

Al Fondo de Investigación y Desarrollo para la Competitividad-FIDECOM-INOVATE PERÚ, por financiar el presente proyecto a través de la Asociación de Productores Conservacionistas de Molinopampa-APROCOM, y al Instituto de Investigación para el Desarrollo Sustentable de Ceja de Selva (INDES CES) de la Universidad Nacional Toribio Rodríguez de Mendoza de Amazonas, Perú.

\section{Referencias}

Alegre, J., Sánchez, Y., Pizarro, D., \& Gómez, C. (2019). Manejo de los suelos con sistemas silvopastoriles en las regiones de Amazonas y San Martín. Universidad Nacional Agraria La Molina - Programa Nacional de Innovación Agraria.

AOAC. (1990). Official Methods of Analysis. 15 th ed. Vol 1. Washington, USA.

Bakker, J., \& Wilson, S. (2001). Competitive abilities of introduced and native grasses. Plant Ecology, 157, 117-125. doi: 10.1023 / a: 1013972403293

Boschini, C. (2006). Nutrientes digeribles, energía neta y fracciones proteicas de la morera (Morus alba) aprovechables en vacas lecheras. Agronomía Mesoamericana, 17(2), 141-150. doi: 10.15517/am.vl7i2.5154 
Castro, R., Hernández, A., Ramírez, O., Aguilar, G., Enríquez, J. F., \& Mendoza, S. I. (2013). Crecimiento en longitud foliar y dinámica de población de tallos de cinco asociaciones de gramíneas y leguminosa bajo pastoreo. Revista Mexicana de Ciencias Pecuarias, 4(2), 201-215. doi: 10.13140/RG.2.1.2879.0885

Clarke, T., Flinn, P.C., \& Mcgowan, A.A. (1982). Low-cost pepsin-cellulase assays for prediction of digestibility of herbage. Grass Forage Sc., 37(2), 147-150. doi: 10.1111/j.1365-2494.1982.tb01590.x

Clavero, T. (2020). Utilización de frutos de árboles forrajeros en la ganadería tropical. Revista De La Universidad Del Zulia, 4 (8), 29-36. Recuperado a partir de https://www.produccioncientificaluz.org/index.php/rluz/article/view/31108

Combatt, E., Jarma, A., \& Paternina, E. (2015). Bromatología de Brachiaria decumbens Stapf y Cynodon nlemfuensis Vanderyst en suelos sulfatados ácidos en Córdoba, Colombia. Revista Mexicana de Ciencias Pecuarias, 6(5), 1035-1049. doi: 10.29312/remexca.v6i5.597

Dresseno, A. L. P., Guido, A., Balogianni, V., \& Overbeck, G. E. (2018). Negative effects of an invasive grass, but not of native grasses, on plant species richness along a cover gradient. Austral Ecology. doi:10.1111/aec.12644

Egan, M., Galvin, N., \& Hennessy, D. (2017). Incorporating white clover (Trifolium repens L.) into perennial ryegrass (Lolium perenne L.) swards receiving varying levels of nitrogen fertilizer: Effects on milk and herbage production. Journal of Dairy Science, 101(4), 1-16. doi: 10.3168/jds.2017-13233

Estupiñán, K., Vasco, D., \& Duchi, N. (2007). Digestibilidad de los componentes de la pared celular del forraje de Canavalia ensiformis (L) DC. en diferentes edades de corte. Revista Tecnológica ESPOL, 20(1), 223-228.

Fernández, J. L., Benítez, D. E., Gómez, I., Cordoví, E., \& Leonard, I. (2001). Dinámica de crecimiento del pasto Brachiaria radicans vc Tanner en las condiciones edafoclimáticas del valle del Cauto en la provincia Granma. Revista Cubana de Ciencias Agrícolas, 35(4), 399-405.

Fernández, J. L., Benítez, D. E., Gómez, I., \& Tandrón, I. (2000). Efecto de la edad de rebrote en el rendimiento de Brachiaria purpurascens vc Aguada en Cuba. Revista Cubana de Ciencias Agrícolas, 34(3), 267-272.

Gandullo, R., Fernández, C., Schmid, P., \& Giménez, G. (2013). Efecto de la variación ambiental en la flora, vegetación y productividad de los humedales "Mallines" de la provincia de Neuquén. Argentina. Revista de la Facultad de Agronomía UNL, 22(2), 75-83. Recuperado de http://rdi.uncoma.edu.ar//handle/123456789/15515

Godfree, R., Firn, J., Johnson, S., Knerr, N., Stol, J., \& Doerr. V. (2017). Why non-native grasses pose a critical emerging threat to biodiversity conservation, habitat connectivity and 
agricultural production in multifunctional rural landscapes. Landscape Ecol., 32, 1219-1242 (2017). doi: 10.1007/s10980-017-0516-9

Godoy, V., Barrera, A., Vivas, R., Quintana, J., Peña, M., Villota, L., Casanova, L., \& Avellaneda, J. (2012). Evaluación fenológica y digestibilidad in vivo de la leguminosa forrajera (Arachispintoi) en diferentes edades de corte. Ciencia y Tecnología, 5(2), 7-16.

IIAP. (2007). Zonificación ecológica y económica del Departamento de Amazonas. 1ª ed. IIAP. Lima, Perú. 199 p.

Lemaire, G., \& Agnusdei, M. (2000). Leaf tissue turnover and efficiency of herbage utilization. En: G Lemaire, J. Hodgson, A. Moraes, P. C. F. Carvalho, \& C. Nabinger (Eds). Grassland ecophysiology and grazing ecology (pp. 265-287). London: CAB International.

Oliva, M., Collazos, R., Vásquez, H., Rubio, K., \& Maicelo, J. L. (2019). Composición florística de especies herbáceas forrajeras en praderas naturales de las principales microcuencas ganaderas de la región Amazonas. Scientia Agropecuaria, 10(1), 109-117. doi: 10.17268/sci.agropecu.2019.01.12

Oliva, M., Oliva, C., Rojas, D., Oliva, M., \& Morales, A. (2015a). Identificación botánica de especies nativas de pastos más importantes de las cuencas lecheras de Molinopampa, Pomacochas y Leymebamba, Amazonas, Perú. Scientia Agropecuaria, 6(2), 125-129. doi: 10.17268/sci.agropecu.2015.02.05

Oliva, M., Rojas, D., Morales, A. Oliva, C. \& Oliva, M. A. (2015b). Contenido nutricional, digestibilidad y rendimiento de biomasa de pastos nativos que predominan en las cuencas ganaderas de Molinopampa, Pomacochas y Leymebamba, Amazonas, Perú. Scientia Agropecuaria, 6(3), 211-215. doi: 10.17268/sci.agropecu.2015.03.07

Pedrini, S., Lewandrowski, W., Stevens, J. C., \& Dixon, K. W. (2018). Optimising seed processing techniques to improve germination and sowability of native grasses for ecological restoration. Plant Biology, 2l(3), 415-524. doi: 10.1111/plb.12885

Pereira-Crespo, S., Valladares, J., Flores, G., Díaz, N., Fernández, B., Resch, C., Gonzales, A., Bande, M. \& Rodríguez, X. (2012). Rendimiento y valor nutritivo de nuevas leguminosas anuales como cultivo de invierno en rotaciones forrajeras intensivas en Galicia. Revista pastos, 42(1), 2950 .

Primavesi, A., \& Primavesi, O. (2002). En Brasil, optimizando las interacciones entre el clima, el suelo, los pastizales y el ganado. Revista Leisa, 18(1), 15-16. Recuperado de http://www.leisaal.org/web/index.php/volumen-18-numero-1/2266-en-brasil-optimizando-las-interaccionesentre-el-clima-el-suelo-los-pastizales-y-el-ganado

Rodríguez, A. G., Patiño, P. R., Altahona, B. L., \& Gil, B. J. (2011). Dinámica de crecimiento de pasturas con manejo rotacional en diferente topografía en un sistema de producción de carne 
vacuna en Córdoba, Colombia. Revista Colombiana de Ciencias Animales, 3(1), 47-61. doi: 10.24188/recia.v3.nl.2011.249

SPSS Inc. Lanzado en 2006. SPSS para Windows, Versión 15.0. Chicago, SPSS Inc.

Uvidia, H., Leonard, I., Benítez, D., \& Buestan, D. (2013). Dinámica del crecimiento de la Maralfalfa (Pennisetum sp.), en condiciones de la Amazonia Ecuatoriana. Revista Amazónica:Ciencia y Tecnología, 2(1), 14-18. Recuperado de https://revistas.proeditio.com/REVISTAMAZONICA/article/view/177

Velasco, M., Hernández, A., Vaquera, H., Martínez, J., Hernández, P., \& Aguirre, J. (2018). Growth analysis of (Panicum maximum Jacq.) Cv. Mombasa. Revista MVZ Córdoba, 23(Supl), 69516963. doi: $10.21897 / \mathrm{rmvz} .1415$

Villalobos, L., \& Arce, J. (2014). Evaluación agronómica y nutricional del pasto estrella africana (Cynodon nlemfuensis) en la zona de Monteverde, Puntarenas, Costa Rica. II. Valor nutricional. Agronomía Costarricense, 38(1), 133-145. Recuperado de http://www.mag.go.cr/rev_agr/v38n01_133.pdf

Wachendorf, M., Collins, R. P., Elgersma, A., Fothergill, M., Frankow-Lindberg, B. E., Ghesquiere, A., ... Connollyi, J. (2001). Overwintering and growing season dynamics of Trifolium repens L. in mixture with Lolium perenne L.: A model approach to plant-environment interactions. Annals of Botany, 88(Special Issue), 683-702. doi: 10.1006/anbo.2001.1496 